\title{
PRIMORDIAL FRACTAL DENSITY PERTURBATIONS AND STRUCTURE FORMATION IN THE UNIVERSE : 1-DIMENSIONAL COLLISIONLESS SHEET MODEL
}

\author{
Takayuki TATEKAWA ${ }^{1}$ and Kei-ichi MAEDA ${ }^{1,2}$ \\ 1 Department of Physics, Waseda University, 3-4-1 Okubo, Shinjuku, Tokyo 169-8555, JAPAN \\ 2 Advanced Research Institute for Science and Engineering, Waseda University, Shinjuku, Tokyo 169-8555, \\ JAPAN \\ E-mail: tatekawa, maeda@gravity.phys.waseda.ac.jp
}

\begin{abstract}
Two-point correlation function of galaxy distribution shows that the structure in the present Universe is scale-free up to a certain scale (at least several tens Mpc), which suggests that a fractal structure may exist. If small primordial density fluctuations have a fractal structure, the present fractal-like nonlinear structure below the horizon scale could be naturally explained. We analyze the time evolution of fractal density perturbations in Einstein-de Sitter universe, and study how the perturbation evolves and what kind of nonlinear structure will come out. We assume a one-dimensional collisionless sheet model with initial Cantor-type fractal perturbations. The nonlinear structure seems to approach some attractor with a unique fractal dimension, which is independent of the fractal dimensions of initial perturbations. A discrete self-similarity in the phase space is also found when the universal nonlinear fractal structure is reached.
\end{abstract}

Subject headings: cosmology: theory — large-scale structure — fractal — 1-dimensional sheet model

\section{INTRODUCTION}

The present Universe shows a variety of structures. The galaxies are not distributed randomly in the Universe. Totsuji \& Kihara (1969) and Peebles (1974) showed that the observed two-point correlation function $\xi(r)$ is given by a power law with respect to a distance $r$ as $\xi(r) \sim r^{-\gamma}$ with $\gamma \sim 1.8$. The recent galaxy surveys also agree with this result, i.e. the power $\gamma$ is nearly equal to 1.8 (CfA (Geller \& Hachra (1989)), LCRS (Jing, Mo, \& Börner (1998)), and ESP (Guzzo et al. (1998, 1999)). This may imply that the present distribution of galaxies is fractal. Sylos Labini, Montuori, \& Pietronero (1998) have also claimed that all available data are consistent with a fractal structure with the dimension $D \sim 2$ up to the deepest observed scale $\left(1000 h^{-1}[\mathrm{Mpc}]\right)$. However, the observation of Cosmic Microwave Background Radiation (CMBR) has revealed that the Universe in the recombination era is homogeneous and isotropic at least in very large scale. Although CMBR observation seems to be more reliable, we should not decide yet whether the large scale structure of the Universe is really fractal up to the horizon scale or not. To answer this question more definitely, we should await forthcoming next galaxy survey projects (Colless (1995), Maddox (1997), Loveday \& Pier (1998), Knapp et al. (1999)).

However, since it seems true that the galaxy distribution is really fractal up to a certain scale, one may ask how such a structure is formed in the evolution of the Universe. One of the most plausible explanations is that the nonlinear dynamics of the perturbations will provide such a scale-free structure during the evolution of the Universe. The pioneering work to explain the power-law behavior in nonlinear stage has 
been done by Davis \& Peebles (1977). They assume a self-similar evolution of density fluctuation and some additional condition, i.e. a physical velocity $\dot{r}$ vanishes in nonlinear regime. Then they showed a relation between the power index $\gamma$ of two-point correlation function and that of initial power spectrum $n$ as $\gamma=3(n+3) /(n+5)$. If we have $n=0$, then we find that $\gamma=1.8$. Since their additional condition is not trivial and might not be appropriate, Padmanabhan (1996) and Yano \& Gouda (1998a) extended their model to the case with non-vanishing $\dot{r}$. They found that the relation between $n$ and $\gamma$ is $\gamma=[3 h(n+3)] /[2+h(n+3)]$, where, $h \equiv-a\langle\dot{x}\rangle / \dot{a} x$, which is a ratio of a peculiar velocity to the Hubble expansion. With this result, $\gamma$ can vary from 0 to 2 for $n=1$ (Harrison-Zel'dovich spectrum) and $0 \leq h \leq 1$ ( $h=1$ corresponds to the Davis-Peebles solution). Since we do not know the stability of those solutions, in order to find which value of $\gamma$ is most likely, we should study the dynamics of density fluctuations in other methods, e.g. $N$-body simulation. Several groups in fact showed that a power-law behavior in two-point correlation function is obtained by $N$-body simulation with appropriate primordial density fluctuations (Miyoshi \& Kihara (1975), Efstathiou (1979), Aarseth, Gott III, \& Turner (1979), Frenk, White, \& Davis (1983), Davis et al. (1985), Jing (1998)).

The question is whether those power-law behaviors mean that we have a fractal structure in the present Universe. Peebles (1985) and Couchman \& Peebles (1998) showed how to proceed with a high resolution analysis in the $N$-body simulation using a kind of renormalization method. They have used Davis-Peebles solution as a scaling relation. Without such an ansatz, we do not know whether usual $N$-body simulation is suitable to discuss the formation of a fractal structure. With the present state of computers, it may not be possible to obtain high enough resolution to analyze a fractal structure.

As for a fractal structure in the Universe, one may ask another question. Did the Universe not have any non-trivial structure such as a fractal in the initial density fluctuations? In the conventional approaches, initial density perturbations are usually assumed to be given by a power-law (or a power-law-like) spectrum with random Gaussian phase. Although such initial conditions may provide the presently observed nonlinear scale-free structure via nonlinear dynamics, no one has shown whether such a structure is fractal or not, and if yes, what kind of fractal structure comes out. To provide a fractal structure in the present Universe, we may adopt an alternative scenario, in which primordial density fluctuations have already a fractal-like structure in the beginning. Note that a background spacetime is assumed to be a smooth universe, which is described by the Einstein-de Sitter universe, but not a fractal universe. The properties of an initial fractal may be preserved during the evolution of the Universe, then nonlinear fractal structure will be formed. In fact, De Gouveia Dal Pino et al. (1995) reported that the temperature fluctuation of CMBR has a fractal relation, and recently, Pando \& Fang (1998) and Feng \& Fang (2000) also reported that non-Gaussianity was detected in the distribution of Ly $\alpha$ forest lines in the QSO absorption spectra. In this scenario, several natural questions may arise. How does such a primordial fractal perturbation evolve into nonlinear regime? Will any properties of the initial fractal be preserved during the evolution of the Universe, or not? If not, what kind of nonlinear structure will come out at present? Is there any fundamental difference in the structure formation process between a conventional density perturbation and the present fractal one? In order to answer those questions, we study the time evolution of the initial density fluctuations with a fractal structure in Einstein de-Sitter universe.

Since we are interested in a fractal structure, a quite high resolution is required in our calculation. As we discussed, $N$-body simulation may not have enough resolution in the present state of computer development, unless we develop some skillful method. So, in this paper, we consider only a very simple toy model, which is a one-dimensional (1-D) sheet model, in order to get some insight into the questions raised in the above. To set up primordial fractal density perturbations, we distribute $N$ sheets initially by 
some systematic rule, i.e. we apply a Cantor set or random Cantor-type set (see below). Mathematically, in order to construct a Cantor set, the procedure must be repeated an infinite number of times, but it is not practically possible to set up such initial data. We therefore stop the procedure at a certain point, i.e. the initial set is given by several times removing line segments with a given ratio (Falconer (1990)). This could be justified because an infinite scale-free structure never exists in the real Universe. In order to construct the initial density perturbations, we set that the remaining segments have small positive density perturbations, while the removed ones correspond to small negative ones. Since we study a 1-D sheet-model, the motion of each sheet is described by an analytic solution (Zel'dovich (1970)), which guarantees enough resolution to analyze a fractal structure.

In $\S 2$, we present our formalism and initial setting. As for the initial data, we consider three cases: regular Cantor set, random Cantor-type set, and random white noise. Comparing those time evolutions, we show our results in $\S 3$. In $\S 4$, we focus particularly on the phase space. The conclusion and discussion follow in $\S 5$.

\section{FORMALISM AND INITIAL DATA}

\subsection{Dynamical Equations}

In order to study the structure formation of the expanding Universe, there are so far three approaches: $N$-body simulation, the Eulerian perturbation approach and the Lagrangian one. Although the final answer for structure formation would be obtained by $N$-body simulation, it may not be possible to so far answer the questions about a fractal structure. As for the perturbation approaches, these are just an approximation and will break down in nonlinear regime, although the Lagrangian approach would be better if we are interested in the density perturbations. This is just because a density fluctuation $\delta$ and a peculiar velocity $\mathbf{v}$ are perturbed quantities in the Eulerian approach (Peebles (1980)), while displacement of particles is assumed to be small in the Lagrangian approach (Zel'dovich (1970), Bouchet (1992, 1995), Coles \& Lucchin (1995), Catelan (1995)). Its first order solution is the so-called Zel'dovich approximation (Zel'dovich (1970)). The Lagrangian approach is confirmed to be better than the Eulerian approach by comparison of these results in several cases (Munshi, Sahni, \& Starobinsky (1994), Sahni \& Shandarin (1996), Yoshisato, Matsubara, \& Morikawa (1998)).

The perturbation variable $\mathbf{S}$ in the Lagrangian approach describes a displacement of dust particles from a uniform distribution and is defined as:

$$
\begin{aligned}
& \mathbf{x}=\mathbf{q}+\mathbf{S}(q, t) \\
& \mathbf{x}: \text { Eulerian comoving coordinate, } \\
& \mathbf{q}: \text { Lagrangian comoving coordinate } .
\end{aligned}
$$

The density fluctuation is given by the Jacobian $J$ as

$$
\delta(\mathbf{q}, t)=\frac{1-J}{J}
$$

where $J \equiv \operatorname{det}(\partial \mathbf{x} / \partial \mathbf{q})$.

Even though the Lagrangian approach is better, it is still an approximation and then is not suitable to discuss a highly nonlinear structure formation. However, there is one exceptional case. If the distribution 
is plane symmetric, the system is one-dimensional, and then the Zel'dovich approximation turns out to be an exact solution. Hereafter, we discuss only one-dimensional problem, which Lagrangian perturbation is given by

$$
x=q+S(q, t)
$$

where $x$ and $q$ are the one-dimensional Eulerian and Lagrangian comoving coordinates, respectively. For Einstein de-Sitter model, the solution is given by (Gouda \& Nakamura (1989), Bouchet et al. (1995)):

$$
S(q, t)=a(t) S_{1}(q)+a(t)^{-3 / 2} S_{2}(q)
$$

where the scale factor $a$ changes as $a=\left(t / t_{0}\right)^{2 / 3}$. Then we find the position and its velocity of a dust particle at a scale factor $a(t)$ with respect to the Lagrangian coordinate $q$ as

$$
\begin{aligned}
& x(q, a)=q+a(t) S_{1}(q)+a(t)^{-3 / 2} S_{2}(q), \\
& \tilde{v}(q, a)=S_{1}(q)-\frac{3}{2} a(t)^{-5 / 2} S_{2}(q)
\end{aligned}
$$

where we have introduced a new peculiar velocity $\tilde{v} \equiv \partial x / \partial a$. In what follows, we use a scale factor $a(t)$ as a time coordinate instead of the physical time $t$.

Although the Zel'dovich solution is exact, in studying a formation of nonlinear structure, a serious problem will soon arise. As a density fluctuation grows, we find a shell crossing. For a realistic matter fluid, a pressure may prevent such a singularity from forming. Then the solution will no longer describe the evolution of perturbations after a shell crossing. However, we may have another choice. If instead of a usual matter fluid, we have a collisionless particle such as some dark matter, we can go beyond the shell crossing. The particles, which are described by plane parallel sheets, will pass through each other without collision. Then after this crossing, we rediscover that the Zel'dovich solution is again exact. Therefore, we have a series of exact solutions, which is almost analytic (Gouda \& Nakamura (1989), Yano \& Gouda (1998b)). We shall call it the 1-D sheet model.

To be more precise, when a crossing by two sheets has occurred, those two sheets exchange their numbering as follows. Suppose there are two sheets $q_{1}, q_{2}\left(q_{1}<q_{2}\right)$ with the Eulerian coordinates $x\left(q_{1}, a\right), x\left(q_{2}, a\right)\left(x\left(q_{1}, a\right)<x\left(q_{2}, a\right)\right)$ and with velocities $\tilde{v}\left(q_{1}, a\right), \tilde{v}\left(q_{2}, a\right)$, respectively. Assume those sheets cross over at $a_{\text {cross }}$, i.e. $x\left(q_{1}, a_{\text {cross }}\right)=x\left(q_{2}, a_{\text {cross }}\right)$ with $\tilde{v}\left(q_{1}, a_{\text {cross }}\right)>\tilde{v}\left(q_{2}, a_{\text {cross }}\right)$. For the evolution after a shell crossing $\left(a>a_{\text {cross }}\right)$, exchanging their numbering $\left(q_{1} \rightleftarrows q_{2}\right)$ as

$$
\begin{aligned}
& x\left(q_{1}, a_{\text {cross }}\right) \rightarrow x\left(q_{2}, a_{\text {cross }}\right), \quad \tilde{v}\left(q_{1}, a_{\text {cross }}\right) \rightarrow \tilde{v}\left(q_{2}, a_{\text {cross }}\right), \\
& x\left(q_{2}, a_{\text {cross }}\right) \rightarrow x\left(q_{1}, a_{\text {cross }}\right), \quad \tilde{v}\left(q_{2}, a_{\text {cross }}\right) \rightarrow \tilde{v}\left(q_{1}, a_{\text {cross }}\right),
\end{aligned}
$$

we find again a natural ordering between the Lagrangian and Eulerian coordinates, i.e. $x\left(q_{1}, a\right)<x\left(q_{2}, a\right)$ for $q_{1}<q_{2}$. By this exchange, we obtain a new distribution of sheets $(x(q, a), \tilde{v}(q, a))$ just after a shell crossing. Using this distribution as an initial data, we find a next exact time evolution of the system by Zel'dovich solution. In order to fix the initial data, we have to determine $S_{1}(q)$ and $S_{2}(q)$ in () for the given distribution $(x(q, a), \tilde{v}(q, a))$. From (5, 6) we find the solution is

$$
\begin{aligned}
& S_{1}(q)=\frac{3}{5 a}(x-q)+\frac{2}{5} \tilde{v} \\
& S_{2}(q)=\frac{2}{5} a^{3 / 2}\{(x-q)-a \tilde{v}\} .
\end{aligned}
$$


The new exact solution (4) with (8) is valid until we encounter next shell crossing. another two sheets will cross over.

We repeat this prescription every time when we encounter a shell crossing. As a result, we obtain a series of the Zel'dovich's exact solutions, which is regarded as an analytic solution for the 1-D collisionless sheet model. Note that this prescription is still valid in multi-stream region. Using this prescription, Gouda \& Nakamura (1989) investigated a time evolution for the density perturbations with a scale-free initial power spectrum. They showed that the power spectrum will approach some characteristic value independent of the power index of the initial spectrum. This characteristic power index -1 is predicted by a catastrophe theory. Recently, Yano \& Gouda (1998b) investigated a time evolution of the density perturbations for an initial power spectrum with a cutoff. In this case, they found that a self-similarity in all scales is no longer valid. The spectrum is classified into five ranges by its power index. Some spectra coincide with the above one for some scale ranges, but another power index, which is independent of the initial power spectrum, appears just beyond the cut-off scale. In $\S 5$, we show our result comparing with their result.

Since this 1-D sheet model is powerful enough to see the fine structure, we shall use this model to analyze a time evolution of the primordial fractal density perturbations.

\subsection{Setting Up Initial Data}

Since we are interested in initial density fluctuations with a fractal distribution, we have to construct such an initial data. For the sake of simplicity, we apply a Cantor set, or a random Cantor-type set (see below), in our construction. A Cantor set is given by the following procedure. We first divide a line with the length $L$ by some integer $n_{D}$ and then remove one line segment at the center. If $n_{D}$ is an even integer, not dividing a line by $n_{D}$, we just remove a line segment with the length $L / n_{D}$ from the center of the line. We then repeat this procedure for the remained line segments. In mathematical definition, the removal procedure must be repeated infinite times. However we believe that a fractal structure, even if it exists in the Universe, is not a mathematical one but its self-similarity may end at some scale. Then we also stop our procedure after a finite number of repetitions. We regard the remaining line segment as a region of positive density fluctuations $\left(\delta_{+}\right.$region), while the removed part corresponds to a region of negative density fluctuations $\left(\delta_{-}\right.$region $) . \delta_{+}(>0)$ and $\delta_{-}(<0)$ are chosen to be uniform, i.e. both $\delta_{+}$and $\delta_{-}$are some constants in all regions such that $\delta_{+} \simeq\left|\delta_{-}\right| \simeq 10^{-3}$. Although this is very artificial and the realistic perturbations may depend on each scale just like conventional density perturbations, we analyze only this simplest case here (see Discussion). In addition to the Cantor set, in order to see the universality of our results, we also consider a random Cantor-type set as well as a distribution constructed by a white noise. We describe in more detail how to construct the initial data for each case.

\subsubsection{Regular Cantor set}

In this case, we consider seven initial data. Each data is constructed by removing central line segments with a fixed ratio $\left(1 / n_{D}\left(n_{D}=3,6,8,10,12,15,20\right)\right)$ from the remained parts. Each density fluctuation has a different fractal dimension given by

$$
D_{0}=\frac{\log 2}{\log \left[2 n_{D} /\left(n_{D}-1\right)\right]} .
$$


We assume that $\delta_{+} \sim\left|\delta_{-}\right|$. With this ansatz, we have fixed the repetition number of removal procedure $\left(N_{R}\right)$. Although the number $N_{R}$ can be different for each initial data, in order to keep the same resolution for each model, (i.e. for the ratio of the smallest line segment $\ell$ to that of the whole region $L$ (our calculation space) to be almost same), we set $N_{R}=5 \sim 7$. We also fix $\delta_{+}=10^{-3}$, which determines the negative density perturbation $\delta_{-}$such that the mean of fluctuations must vanish (see Table 1 ).

\begin{tabular}{ccccc}
$n_{D}$ & $D_{0}$ & $N_{R}$ & $\ell / L$ & $\delta_{-}$ \\
\hline 3 & 0.631 & 5 & $1 / 243$ & $-0.152 \times 10^{-3}$ \\
6 & 0.792 & 6 & $1 / 191.10$ & $-0.504 \times 10^{-3}$ \\
8 & 0.838 & 7 & $1 / 325.95$ & $-0.647 \times 10^{-3}$ \\
10 & 0.868 & 7 & $1 / 267.62$ & $-0.917 \times 10^{-3}$ \\
12 & 0.888 & 7 & $1 / 235.36$ & $-1.19 \times 10^{-3}$ \\
15 & 0.909 & 7 & $1 / 207.47$ & $-1.61 \times 10^{-3}$ \\
20 & 0.931 & 7 & $1 / 183.29$ & $-2.31 \times 10^{-3}$
\end{tabular}

Table 1 :

The number of division $n_{D}$, its fractal dimension $D_{0}$, the repetition number of removal procedure $N_{R}$, the resolution (the ratio of the length of the shortest line segment to that of the whole system $\ell / L$ ), and each negative density perturbation $\delta_{-}$.

We show one example of initial data for $n_{D}=10$ in Fig. 1 . We put $2^{17}$ sheets in our calculation space $L$. By use of a box-counting method (the box length $x$ is chosen from $2^{-16} L\left(\sim 1.5 \times 10^{-5} L\right)$ to $10^{-1} L$ ), we have checked the fractal dimension of initial fluctuations. We calculate the number $N(x)$ of boxes with a length $x$ in which $\delta_{+}$-segments exist. The result is shown in Fig. 2. We find a power law in the $x-N(x)$ relation for the range of $10^{-3} L \leq x \leq 10^{-1} \mathrm{~L}$. From this curve, we estimate the fractal dimension as 0.868 , which is the same value as that of the present Cantor set. Below $x=10^{-3} L$, we have a small deviation from a power law relation, which corresponds to the limit of the resolution of our present model.

Since the Cantor set is quite systematically constructed, one may wonder that our results strongly depend on such a special setting and may not be universal. To answer such a question, we also analyze two different initial data settings: one is a random Cantor-type set and the other is just a white noise.

\subsubsection{Random Cantor-type set}

The random Cantor-type set is defined so that the division number $n_{D}$ is fixed as that of a regular Cantor set, while its removal positions of line segments are determined by a random number. According to the box-counting method, we find that the fractal dimension of initial fluctuations is almost the same as that of a regular Cantor set for the same $n_{D}$. In this paper, we analyze 3 random Cantor-type set models: two models with $n_{D}=10$ and one with $n_{D}=12$. One initial distribution for $n_{D}=10$ is shown in Fig. 3 . 


\subsubsection{White Noise}

In order to see whether or not our primordial fractal fluctuations will play an important role in a structure formation, in particular a formation of fractal structure in a nonlinear regime, we also study a time evolution of primordial fluctuations with white noise. The distribution of initial density fluctuations is given by a random number between $-10^{-3} \leq \delta \leq 10^{-3}$. We analyze 2 models, for one of which the initial data is shown in Fig. 4.

\section{TIME EVOLUTION OF PRIMORDIAL FRACTAL DENSITY PERTURBATIONS}

In order to see how structures are formed, we have to describe the distribution by the Eulerian coordinate. It is convenient to compare the distribution at each time by use of the comoving coordinate $x$. We set the initial scale factor $a_{0}=1$. Since our initial density fluctuation is $\sim 10^{-3}$, we find a first shell crossing at $a=a_{\text {cross }} \simeq 10^{3}$. We perform our calculation until $a=(2 \sim 3) \times 10^{4}$.

In order to see the detail to resolve a fractal structure, we put $2^{17}$ sheets in our calculation scale $L$. Using a box-counting method, i.e, counting the number of boxes which contain the region with density perturbation $\delta$ larger than 1 , we determine the fractal dimension of the nonlinear structures. The size of the box ranges from $2^{-16} L$ to $10^{-1} L$

We shall present our results for three types of initial data in order.

\subsection{Regular Cantor Set}

First, we show the results for the regular Cantor set with $n_{D}=10$. The time evolution is depicted in

Fig 5. Because we set $\delta_{+} \simeq\left|\delta_{-}\right| \simeq 10^{-3}$, a nonlinear structure appears at $a \simeq 5 \times 10^{2}$ (Fig. 5(a)). Before a shell crossing, the pattern of density fluctuations remains similar to that of initial distributions, although each separation is going to change through the gravitational interaction. We then find a shell crossing at $a=a_{\text {cross }} \sim 10^{3}$ (Fig. 5(b)). After the shell crossing, the trace of initial Cantor set gradually disappears, because of the exchange of the shells by a crossing (Fig. 5(b)-(f)). Although many sheets cross each other, the peculiar velocities do not vanish immediately because of collisionless sheets, and then the pattern of nonlinear structure will change continuously. After sufficient evolution of the nonlinear structure, we find a self-similarity in the structure, which seems to be fractal. In fact, enlarging some regions, we find similar density distributions (Fig. 6).

In order to judge whether such a structure is fractal or not, we use a box-counting method, which gives 
a fractal dimension $D_{F}$. Before a shell crossing, we find that the dimension $D_{F}$ decreases in time from the initial value $D_{0}=0.868$, but the error in the estimation increases with time (Fig. $7($ a)). This is because although the pattern of initial density fluctuations remains even in a nonlinear stage before a shell crossing, change of each separation breaks the initial fractal distribution. Then, the initial fractal distribution seems to disappear.

However, after a shell crossing, the fractal dimension starts to increase again and the error in the estimation becomes much smaller. The fractal structure seems to recover. More surprisingly, the dimension $D_{F}$ approaches some constant $\left(D_{\text {asym }} \sim 0.9\right)$ after $a \simeq 1.5 \times 10^{4}$, which is a little bit different from the initial fractal dimension $D_{0}=0.868$ (Fig. 7.(b)). In fact, $D_{F}=0.889 \pm 0.009$ at $a=1.5 \times 10^{4}$ and $D_{F}=0.890 \pm 0.002$ at $a=2 \times 10^{4}$. Although $D_{0}=0.868$ is out of the error bar of $D_{F}$, the difference is very small. However we will see that $D_{F}$ is really independent of the initial fractal dimension $D_{0}$ later in the case of different initial distributions (see Fig. 10).

We also calculate the two-point spatial correlation function of nonlinear structures. If the structure is fractal, we have a relation between a fractal dimension and a power index of the correlation function (Falconer (1990)). The correlation function here is evaluated for the nonlinear regions where the density fluctuation $\delta$ becomes larger than 1 . At $a=10^{3}$, just after a first shell crossing, the correlation function shows a rapid oscillation between a positive and a negative values because of the periodic pattern of the Cantor set. After a shell crossing, the pattern of Cantor set disappears, and then such an oscillation also vanishes. After enough time passes, i.e. when the stable fractal structure is found, the correlation function becomes also stable (Fig. 8). The function is positive for the distance of $x \leq 5 \times 10^{-2} L$, beyond which it becomes negative because a shell crossing does not yet occur for such a scale and the largest nonlinear structure is about $5 \times 10^{-2} \mathrm{~L}$. The trace of initial Cantor set still remains. If the structure is fractal, the correlation function must show a power-law behavior. In fact, from Fig. 8, we find a power law relation in the range of $10^{-4} L<x<10^{-2} L$ as $\xi \propto x^{-\gamma}$ with $\gamma=0.130 \pm 0.005$. When the fractal dimension is $D_{F}$, and the correlation function is given as

$$
\xi(x) \propto x^{-\left(1-D_{F}\right)}
$$

then we find $\gamma=1-D_{F}$. The evaluated fractal dimension from two point correlation is $D_{F}=0.870 \pm 0.005$ at $a=10000$, which is close to the value obtained by a box-counting $D_{F}=0.862 \pm 0.009$. We show the time evolution of two "fractal" dimensions $D_{F}$ obtained from two point correlation and by a box-counting method in Fig. 9. From Fig. 9, we confirm that the power index $\gamma$ is well correlated to the fractal dimension $D_{F}$.

We find a fractal structure after a shell crossing. The fractal dimension is close to the fractal 
dimension of the initial density distributions. Does this dimension reflect that of the initial distributions? If this is so, why does it disappear once around a first shell crossing time and recover at very late time? In order to answer these question, we have looked for other initial conditions with different $n_{D}$ s, i.e. $n_{D}=3,6,8,12,15,20$. The fractal dimensions $D_{0}$ of the initial density distributions are given in Table 1. As for the time evolution, we find similar behaviour for all models. The evolution of "fractal" dimension $D_{F}$ is shown in Fig. 10. Surprisingly, for all models, all $D_{F}$ approach about 0.9 at $a=(2 \sim 3) \times 10^{4}$, which is the end of our calculation. To confirm our result, we have checked the size-number $(x-N(x))$ relation by a box-counting method, which shows almost a straight line as in Fig. 2. Since the initial dimensions of primordial fluctuations were different, we would conclude that the fractal dimension obtained after nonlinear evolution is universal within our numerical accuracy.

\subsection{Random Cantor-type Set}

Since the Cantor set is highly systematically constructed, one may ask whether the present result strongly depends on such a special initial setting. Is the universal dimension of nonlinear fractal structures due to the primordial density fluctuations defined by the regular Cantor set? In order to answer this question, we shall analyze a different model with randomness, which we call a random Cantor-type set defined in the previous subsection.

We analyze 3 models: 2 models with $n_{D}=10$ (model 1 and model 2 ) and 1 model with $n_{D}=12$. We find that just as in the case of regular Cantor sets, the fractal dimension for nonlinear structures always approaches about 0.9 (Fig. 11). We have also checked the size-number $(x-N(x))$ relation in a box-counting method, finding the same result as in the case of a regular Cantor set. Because we remove a line segment at a random position, we usually expect that the smallest segment is smaller than that of the regular Cantor set as we show in the previous subsection. As a result, the stable fractal structure will be formed later compared with the case with a regular Cantor set. In fact, in the case of $n_{D}=12$, we find the stable dimension (0.9) around $a \simeq 2.3 \times 10^{4}$ (Fig.11(c)).

\subsection{White noise case}

Then one may have another question. Is the universal fractal dimension obtained above via nonlinear dynamics and independent of the initial distribution? In order to answer this question, we also analyze a model with white noise fluctuation. We analyze two models. Both models do not show up the above universal fractal dimension, although we find some different stable asymptotic dimension $(\sim 0.7)$ (Fig. 12). The error in estimating the dimension is larger than that of the Cantor set model, and the box-counting 
shows some deviation from the power law relation (Fig. 13). Hence, it would not be a fractal. We will discuss this in out Discussion.

\section{ANALYSIS OF PHASE SPACE}

Although the analysis by a box-counting suggests that a nonlinear fractal structure with a universal dimension appears from primordial fractal fluctuations, we may get more information from a detailed study of the nonlinear structures obtained. For this purpose, we analyze our result in a phase space.

We show the time evolution of the structures for the regular Cantor set with $n_{D}=10$ in a phase space. Initially, the sheets distribution in a phase space is given by a notched curve because $\delta_{+}$and $\delta_{-}$are constant (Fig. 14). If we enlarge the pictures, we find a similar notched curve because of the present initial setting. These notches reflect a self-similarity in the initial Cantor set. This behavior does not change before a shell crossing (Figs. 14 (a), (b)). Only the slopes of the line segments become steeper because of the concentration of sheets. After a shell crossing, the curve in a phase space shows very complicated behavior. Two sheets exchanged by a shell crossing are decelerated by the mutual gravitational interaction, and then the curve will swirl (Gouda \& Nakamura 1989). As the structure evolves, some vortices are combined and form a larger vortex (Fig. 14). When the "fractal" dimension becomes stable around 0.9, we find that the large vortex consists of some similar small vortices. These small vortices also consist of similar but much smaller vortices (Fig. 15). This discrete self-similarity in a phase space is found in all models. As the structure evolves, some vortices are combined and form a larger vortex (Fig. 14). Although the initial fractal distribution seems to disappear, some trace remains in the phase space.

One may wonder why the centers of the vortices appear in $\tilde{v}>0$ region ( or $\tilde{v}<0$ region). The formation of a vortex in a phase space can be easily understood in the case of a single wave $\left(S_{1}=\epsilon \sin q\right)$ (see Gouda \& Nakamura (1989)). In that case, the velocity at the center of the vortex vanishes. Then the appearance of the vortex in $\tilde{v}>0$ region ( or $\tilde{v}>0$ region) seems inconsistent. If we pursue each particle motion, nothing strange happens. They move without swirling except at $\tilde{v}=0$ point as shown in Fig. 16.

As was the case with a regular Cantor-type set, we also find similar results in the phase space (Fig. 17). However, a discrete self-similarity is hard to find, although the larger vortices contain smaller vortices as in the case of the regular Cantor-type set. In the case with white noise fluctuation, we cannot find any hierarchical vortex structure in a phase space (Fig. 18). 


\section{CONCLUSIONS AND DISCUSSION}

We have studied the nonlinear evolution of primordial fractal fluctuations by using a 1-D sheet model. We have analyzed 7 models with initial fluctuations constructed by a regular Cantor set, 3 models with initial fluctuations constructed by a random Cantor-type set, and 2 models with white noise fluctuations. For all models except for the case with white noise, we find a kind of attractor with a universal fractal dimension $(\sim 0.9)$ as the fluctuations evolve into nonlinear regime. In the case with white noise fluctuations, the estimated dimension becomes stable around 0.7 , but the error in the estimation is larger than the other cases and the power-law behavior in a box-counting is also not completely fitted. Then, it may not contain a fractal structure. From the phase space analysis, we find a hierarchical structure, that is, the large vortex consists of some similar small vortices, and such small vortices again consist of similar but much smaller vortices. In particular, we find a discrete self-similarity for the model with a regular Cantor set.

Why is the fractal dimension close to 0.9 ? Is it really universal? Is the present fractal structure really an attractor? Although we need more analysis to answer this question, we have some hints in previous work. Gouda and Nakamura studied the present 1-D sheet model for the initial power law spectrum. They found two types of generic singularities when we have a shell crossing (Gouda \& Nakamura 1988, 1989). When a first shell crossing appears, the relation between Eulerian and Lagrangian coordinates must be

$$
x=q_{c}+\beta\left(q-q_{c}\right)^{3}+\cdots,
$$

while that after a shell crossing turns out to be

$$
x=q_{c}+\beta\left(q-q_{c}\right)^{2}+\cdots .
$$

Following Arnold's classification, the former and latter cases are classified into A3 and A2, respectively. A3 is structurally unstable and may appear transiently in the expanding Universe. A2 is structurally stable and appears universally for the initial power-law spectrum. The latter case gives

$$
\begin{aligned}
\delta_{k} & =\int \delta(x) e^{i k x} d x \\
& \propto \int(\beta x)^{-1 / 2} e^{i k x} d x \\
& =(\beta k)^{-1 / 2} \int \eta^{-1 / 2} e^{i \eta} d \eta,
\end{aligned}
$$

i.e. $P(k) \sim k^{-1}$. This predicts $\gamma=0$, i.e. $D_{F}=1$, which is rather close to our "universal" dimension 0.9. Although one may wonder that these are essentially the same, we have another result which suggests that there seems to exist a new type of stable phase. Recently, Yano and Gouda analyzed a more realistic case, i.e. the initial power law spectrum with a cut-off and found 5 characteristic regions in Fourier space (Yano \& 
Gouda 1998a, b). The regime 1 is the linear one and then it is just an initial power spectrum. In the regime 2, they found $P(k) \sim k^{-1}$, which is the single-caustic regime (Gouda \& Nakamura (1989)). The regime 3 is called the multi-caustic regime, in which the power spectrum depends on the initial power-law index. Beyond the cut-off scale, two regimes appear, one gives $P(k) \sim k^{-1}$ (regime 5 ), which may correspond to A2 type stable solution. In the intermediate wave number $k$ between the regime 3 and regime 5 , they find $k^{\nu}$, which $\nu$ is independent of initial power index and close to 1 , but a little less. They called it the virialized regime. This seems to be a new transient region, which may appear in some specific initial conditions. We would conjecture that the fractal structure with a universal dimension 0.9 corresponds to this virialized regime (regime 4) and the dimension 0.7 found in the case with white noise would be the regime 3 . By reanalyzing the Yano-Gauda model in the case of $k=0$, we have confirmed that $\nu=\sim 0.9$. We also find a small tail with index 0.7 in the size-number relation in the Cantor set model with $n_{D}=15,20$ (Fig. 19).

This conjecture is also supported by the analysis for a self-gravitating 1-D sheet model without the background expansion of the Universe (Tsuchiya, Konishi \& Gouda 1994). They found two time scales; one is a micro relaxation time $\left(t_{\text {micro }}=N t_{c}\right)$ and the other is a global relaxation time $\left(t_{\text {global }}=4 \times 10^{4} N t_{c}\right)$, where $t_{c}=\sqrt{L / 4 \pi G N m}$ is a crossing time. After $t_{\text {micro }}$, some equilibrium state is reached by exchanging particle energy, but the global relaxation is not achieved, i.e. the partition function is not yet described by an equilibrium state such as an ergodic state (Tsuchiya, Konishi \& Gouda 1994). In the present model, we can speculate that the fractal structure is obtained after this micro relaxation time but before the global relaxation time. In fact, if we estimate the above time scales in the present models, we find that $t_{\text {micro }}$ corresponds to $a=5 \times 10^{3}$, while $t_{\text {global }}$ corresponds to $a=5 \times 10^{6}$. The time when we find a stable fractal structure $\left(a=(1 \sim 3) \times 10^{4}\right)$ is between those two time scales. If this speculation is true, our fractal structure is temporal. In the future of the Universe, it will evolve into more relaxed and ergodic state.

Since we analyze the simplest case, we have to extend our analysis to more generic cases. First, we should study different types of fractal in order to check whether the present results are universal for any fractal distributions or not. Secondly, we need to analyze the case with scale-dependent fluctuations. In the present analysis, we set $\delta_{+}=$constant and $\delta_{-}=$constant. In the realistic case, there must be a scale dependence to the fluctuations. In the conventional perturbations, we usually assume a power-law spectrum with some cutoff. Even if the primordial fluctuations contain a fractal structure, their amplitude may depend on the scale. Its dependence may change the present results. In particular, in the present model, the scenario of structure formation could be different from either TOP-DOWN or BOTTOM-UP for some scale-dependence. The primordial fractal fluctuations will evolve directly into a hierarchical nonlinear structure. But, it will definitely depend on the scale dependence of the fluctuations. Secondly, we need to extend the present analysis to other cosmological models, i.e. the open Universe model and $\Lambda \neq 0$ flat 
Universe model. For the 1-D sheet model, the solutions are still exact, and the growth and decay rates in these models are different from those in the Einstein-de Sitter Universe model. We expect that the structure formation after a shell crossing is not the same as that in the present cosmological model, and then the fractal dimension would be different.

For more realistic cases, we must study either the 2-D or 3-D model. Since the Zel'dovich solution is no longer exact, we have to explore a new method. In order to preserve a high resolution, we may develop a kind of renormalization method in $N$-body simulation as Couchman \& Peebles (1998).

Finally, it would also be interesting to look for the origin of such a primordial fractal density perturbation. The inflationary scenario may provide the origin of primordial fluctuations. One may wonder whether such a fractal primordial fluctuation is expected in some inflationary models. If we have more than two scalar fields, then the system is not integrable and may show a chaotic behavior or a fractal property (Easther \& Maeda (1999)). Such a model might show up a kind of fractal density perturbation.

We would like to thank N. Gouda, P. Haines, O. Iguchi, T. Kurokawa, V. Lukash, M. Morikawa, A. Nakamichi, Y. Sota, T. Yano, and A. Yoshisato for many useful discussions. Part of this work was done while KM was participating the program, "Structure Formation in the Universe", at the Newton Institute, University of Cambridge. KM is grateful to the Newton Institute for their hospitality. Our numerical computation was carried out by Yukawa Institute Computer Faculty. This work was supported partially by a Grant-in-Aid for Scientific Research Fund of the Ministry of Education, Science and Culture (Specially Promoted Research No. 08102010), and by the Waseda University Grant for Special Research Projects. 


\section{REFERENCES}

Aarseth S.J., Gott III, J.R., Turner E.L. 1979, ApJ, 228, 664.

Bouchet, F.R., Juszkiewicz, R., Colombi, S., \& Pellat, R. 1992, ApJ, 394, 5.

Bouchet, F.R., Colombi, S.,Hivon, E., \& Juszkiewicz, R. 1995, A\&A, 296, 575B.

Catelan, P. 1995, MNRAS, 276, 115.

Coles, P. \& Lucchin, F. 1995, Cosmology (John Wiley \& Sons).

Colless M.M. 1995, in the Proceedings of "Peculiar Velocities in the Universe", Coral Sea Cosmology

Conference I, Heron Island Maddox, S. 1997, in the Proceedings of Potsdam Cosmology Workshop, "Large Scale Structure: Tracks and Traces" Sept. 15-19, 1997

Couchman, H.M.P. \& Peebles, P.J.E. 1998, ApJ, 497, 499.

Davis, M., Efstathiou, G., Frenk, C.S., \& White, S.D.M. 1985, ApJ, 292, 371.

Davis, M. \& Peebles, P.J.E 1977, ApJS, 34, 425.

de Gouveia Dal Pino, E.M., Hetem, A., Horvath, J.E., de Souza, C.A.W, Villela, T., \& de Araujo, J.C.N. 1995, ApJ, 442, 45.

Easther, R. \& Maeda, K. 1999, Class. Quantum Grav., 16, 1637.

Efstathiou, G. 1979, MNRAS, 187, 117.

Falconer, K. 1990, Fractal Geometry (John Wiley \& Sons).

Feng, L. \& Fang, L.Z. 2000, preprint (astro-ph/0001348).

Frenk, C.S., White, S.D.M., \& Davis, M. 1983, ApJ, 217, 417.

Geller, M.J. \& Huchra, J.P. 1989, Science, 246, 897.

Gouda, N. \& Nakamura, T. 1988, Prog.Theor.Phys., 79, 765.

Gouda, N. \& Nakamura, T. 1989, Prog.Theor.Phys., 81, 633.

Guzzo, L., Bartlett, J.G., Cappi, A. et al. (The ESP Team) 1998, Proceedings of XIV IAP Colloquium

"Wide Field Surveys in Cosmology" (eds. S. Colombi \& Y. Mellier, Paris, May 26-30 1998).

Guzzo, L. et al. 1999, preprint (astro-ph/9901378).

Jing, Y.P., Mo, H.J., \& Börner,G. 1998, ApJ, 494, 1.

Jing, Y.P. 1998, ApJ, 503, L9.

Knapp, J. et al., 1999 The Sloan Digital Sky Survey Project Book http://www.astro.princeton.edu/PBOOK/welcome.htm

Loveday, J. \& Pier, J. 1998, preprint(astro-ph/9809179).

Maddox, S., Proceedings of Potsdam Cosmology Workshop, "Large Scale Structure: Tracks and Traces" Sept. 15-19, 1997.

Miyoshi, K. \& Kihara, T. 1975, Pub. Astron. Soc. Japan, 27, 333.

Munshi, D., Sahni, V. \& Starobinsky, A.A. 1994, ApJ, 436, 517

Padmanabhan, T. 1996, MNRAS, 278, L29

Pando, J. \& Fang, L.Z. 1998, A\&A, 340, 335

Peebles, P.J.E. 1974, A\&A, 32, 197

Peebles, P.J.E. 1980, The Large Scale Structure of the Universe (Princeton University Press).

Peebles, P.J.E. 1985, ApJ, 297, 350 
Sahni, V. \& Shandarin, S. 1996, MNRAS, 282, 641

Sylos Labini, F., Montuori, M., \& Pietronero, L. 1998, Phys.Rep., 293, 61.

Totsuji, H. \& Kihara, T. 1969, Pub.Astron.Soc.Japan, 21, 221.

Tsuchiya, T. , Konishi, T. \& Gouda, N., 1994, Phys. Rev. E, 50, 2607.

Yano, T. \& Gouda, N. 1998a, ApJ, 495, 533.

Yano, T. \& Gouda, N. 1998b, ApJS, 118, 267.

Yoshisato, A., Matsubara, T., \& Morikawa, M. 1998, ApJ, 498, 49 .

Zel'dovich, Ya.B. 1970, A\&A, 5, 84. 


\section{Figure Captions:}

Fig.1. The initial fractal density fluctuation for the regular Cantor set with $n_{D}=10$. Enlarging the picture, we find the same pattern up to the repetition number of removal procedure $N_{R}=7$.

Fig. 2. The size-number $(x-N(x))$ relation in a box-counting method for the model in Fig. 1. The dotted line shows $N(x) \propto x^{-0.868}$. We find that the similarity exists in the range between $10^{-3} \mathrm{~L}$ and $10^{-1} \mathrm{~L}$. Below $x=10^{-3} L$, we have a deviation from the power law relation, which corresponds to our resolution limit $(\ell / L \sim 1 / 267.62)$.

Fig. 3.(a) The initial fractal density fluctuation for the random Cantor-type set with $n_{D}=10$. The random Cantor-type set is defined so that the division number $n_{D}$ is fixed as that of a regular Cantor set, while its removal positions of line segments are determined by a random number. This is called the model 1 in the text. Enlarging the picture, we find the similar pattern up to the repetition number of removal procedure $N_{R}=7$. The fractal dimension of this initial fluctuation is 0.868 , which is the same as that of the regular Cantor set with $n_{D}=10$.

(b) Another initial fractal density fluctuation set up by the same prescription as (a) (model 2).

Fig. 4.(a) The initial density fluctuation created by a white noise. This is called the model 1 in the text.

(b) Another initial density fluctuation set up by the same prescription as (a) (model 2).

Fig. 5.(a) Time evolution of density fluctuation for the case with $n_{D}=10$. This snap shot is at $a=5 \times 10^{2}$, when the positive fluctuation $\delta_{+}$grows just to a nonlinear scale $\left(\delta_{+}=1\right)$.

(b) The same as (a) but at $a \sim a_{\text {cross }} \sim 10^{3}$, which is just after a first shell crossing.

(c) The same as (a) but at $a=6 \times 10^{3}$.

(d) The same as (a) but at $a=10^{4}$.

(e) The same as (a) but at $a=1.5 \times 10^{4}$.

(f) The same as (a) but at $a=2 \times 10^{4}$.

Fig. 6. Hierarchical nonlinear structure at $a=10^{4}$. Enlarging the picture, we find the self-similar structure.

Fig. 7.(a) The evolution of the "fractal" dimension $D_{F}$ of nonlinear structure for the case of $n_{D}=10$. Before a shell crossing $\left(a<a_{\text {cross }} \sim 10^{3}\right)$.

(b) The same as (a) but after a shell crossing $\left(a \geq a_{\text {cross }} \sim 10^{3}\right)$.

Fig. 8. Two-point correlation function of nonlinear structures at $a=10^{4}$. The dotted line is $\xi \propto x^{-0.130}$.

Fig. 9. The time evolution of the "fractal" dimensions $D_{F}$ obtained from two point correlation and by a box-counting method The circles denote $D_{F}$ by two-point correlation $\left(D_{F}=1-\gamma\right)$, while the squares are those produced by a box-counting. Two methods to determine the fractal dimension agree well each other.

Fig. 10.(a) The time evolution of the "fractal" dimension $D_{F}$ of nonlinear structure for $n_{D}=3$.

(b) The same as (a) but $n_{D}=6$.

(c) The same as (a) but $n_{D}=8$.

(d) The same as (a) but $n_{D}=12$.

(e) The same as (a) but $n_{D}=15$.

(f) The same as (a) but $n_{D}=20$. 
Fig. 11.(a) The evolution of the "fractal" dimension $D_{F}$ of nonlinear structure in the case of the random Cantor-type initial fluctuations for $n_{D}=10$ (model 1). The dimension approaches about 0.9 at $a=2 \times 10^{4}$.

(b) The same as (a) $\left(n_{D}=10\right.$, model 2$)$. The dimension approaches about 0.9 at $a=2.8 \times 10^{4}$.

(c) The same as (a) $\left(n_{D}=12\right)$. The dimension approaches about 0.9 after $a=2.3 \times 10^{4}$.

Fig. 12.(a) The evolution of the "fractal" dimension $D_{F}$ of nonlinear structure in the case with white noise fluctuations (model 1). The dimension approaches about 0.7 .

(b) The same as (a) but of model 2. We also find the same stable dimension 0.7.

Fig. 13. The size-number $(x-N(x))$ relations in a box-counting method for the models in Fig. 12. There is some small deviation from a power-law behaviour.

Fig.14.(a) Structure Formation in phase space $\left(n_{D}=10\right)$. At $a=1$. The distribution draws a self-similar graph, because initial density fluctuation was given by a regular Cantor set.

(b) The same as (a) but at $a=10^{3}$. At immediately after shell crossing, the sheets just began production of vortices.

(c) The same as (a) but at $a=3 \times 10^{3}$. The self similarity of vortices exists during four steps.

(d) The same as (a) but at $a=5 \times 10^{3}$. The self similarity of vortices exists during five steps.

(e) The same as (a) but at $a=10^{4}$. Two of $L$ scale structure was begun to combined.

(f) The same as (a) but at $a=1.5 \times 10^{4}$.

(g) The same as (a) but at $a=2 \times 10^{4}$.

Fig. 15. A sheet distribution at $a=2 \times 10^{4}$ in phase space $\left(n_{D}=10\right)$. The dotted squared regions are enlarged in the next figures shown by arrows. We find a discrete self similarity, i.e. a large vortex consists of some similar small vortices, and those small vortices also consist of similar but much smaller vortices.

Fig. 16. The orbits of 7 particles in phase space $\left(n_{D}=10,6000 \leq a \leq 20000\right)$. Each particle did not swirl, however distribution of particles become to set of vortices.

Fig. 17. A sheet distribution at $a=30000$ in phase space for the model with a random Cantor-type set $\left(n_{D}=12\right)$. In this figure, the larger vortices contain smaller vortices as the case of regular Cantor set, but a discrete self-similarity is hard to be found.

Fig. 18. A sheet distribution $a=30000$ in phase space for the model with white noise fluctuations (model 1 ). The vortices were widely spread and a nest of vortices is rarely found.

Fig. 19. The size-number $(x-N(x))$ relation in a box-counting method for the regular Cantor set with $n_{D}=15$ at $a=30000$. The dotted line denotes $N(x) \propto x^{-0.9}$, while the dotted-dash line is $N(x) \propto x^{-0.7}$. 
This figure "fig01.jpg" is available in "jpg" format from: http://arxiv.org/ps/astro-ph/0003124v5 
This figure "fig02.jpg" is available in "jpg" format from: http://arxiv.org/ps/astro-ph/0003124v5 
This figure "fig03.jpg" is available in "jpg" format from: http://arxiv.org/ps/astro-ph/0003124v5 
This figure "fig04.jpg" is available in "jpg" format from: http://arxiv.org/ps/astro-ph/0003124v5 
This figure "fig05.jpg" is available in "jpg" format from: http://arxiv.org/ps/astro-ph/0003124v5 
This figure "fig06.jpg" is available in "jpg" format from: http://arxiv.org/ps/astro-ph/0003124v5 
This figure "fig07.jpg" is available in "jpg" format from: http://arxiv.org/ps/astro-ph/0003124v5 
This figure "fig08.jpg" is available in "jpg" format from: http://arxiv.org/ps/astro-ph/0003124v5 
This figure "fig09.jpg" is available in "jpg" format from: http://arxiv.org/ps/astro-ph/0003124v5 
This figure "fig10.jpg" is available in "jpg" format from: http://arxiv.org/ps/astro-ph/0003124v5 
This figure "fig11.jpg" is available in "jpg" format from: http://arxiv.org/ps/astro-ph/0003124v5 
This figure "fig12.jpg" is available in "jpg" format from: http://arxiv.org/ps/astro-ph/0003124v5 
This figure "fig13.jpg" is available in "jpg" format from: http://arxiv.org/ps/astro-ph/0003124v5 
This figure "fig14.jpg" is available in "jpg" format from: http://arxiv.org/ps/astro-ph/0003124v5 
This figure "fig15.jpg" is available in "jpg" format from: http://arxiv.org/ps/astro-ph/0003124v5 
This figure "fig16.jpg" is available in "jpg" format from: http://arxiv.org/ps/astro-ph/0003124v5 
This figure "fig17.jpg" is available in "jpg" format from: http://arxiv.org/ps/astro-ph/0003124v5 
This figure "fig18.jpg" is available in "jpg" format from: http://arxiv.org/ps/astro-ph/0003124v5 
This figure "fig19.jpg" is available in "jpg" format from: http://arxiv.org/ps/astro-ph/0003124v5 